PROCEEDINGS OF THE

AMERICAN MATHEMATICAL SOCIETY

Volume 135, Number 11, November 2007, Pages 3521-3523

S 0002-9939(07)09010-7

Article electronically published on July 27, 2007

\title{
TWO COUNTEREXAMPLES IN NORMALIZATION
}

\author{
SHREERAM S. ABHYANKAR AND BEN KRAVITZ \\ (Communicated by Bernd Ulrich)
}

\begin{abstract}
In this paper, we offer a correction to Commutative Algebra by Oscar Zariski and Pierre Samuel by noting two erroneous theorems and offering counterexamples for each.
\end{abstract}

\section{INTRODUCTION}

Since the concentration of this paper is on normalization, we find it useful to introduce some notation. Given a $\operatorname{ring} R$ and an overring $S$, we denote the integral closure of $R$ in $S$ by $\bar{R}^{S}$.

In the Commutative Algebra book of Zariski and Samuel [6] at the bottom of page 124, the authors assert that a normalization lemma can be generalized. More specifically, they assert the following:

Claim 1.1. Let $A=R\left[x_{1}, \ldots, x_{n}\right]$ be an integral domain, finitely generated over an infinite domain $R$, and let $d$ be the transcendence degree of the field of quotients of $A$ over the field of quotients $k$ of $R$. Then there exist $d$ linear combinations $y_{1}, \ldots, y_{d}$ of the $x_{i}$ with coefficients in $R$, such that $A$ is integral over $R\left[y_{1}, \ldots, y_{d}\right]$.

This generalized lemma would be true if $R$ were an infinite field, and in fact is proved in 5 (Ch. V, $\S 4$, Theorem 8), but it is not true when $R$ is simply an infinite domain. We will provide a counterexample to this claim in Example 2.1 of the next section, which we take from (E28) on page 592 of Abhyankar's recent book [1].

Claim 1.1 is then used by Zariski and Samuel in the proof of the following claimed theorem on page 125 of $[\underline{6}$ :

Claim 1.2. Let $R$ be a restricted domain, $A=R\left[x_{1}, \ldots, x_{n}\right]$ an integral domain that is finitely generated over $R$, and let $F$ be a finite algebraic extension of the quotient field $k\left(x_{1}, \ldots, x_{n}\right)$ of $A$, where $k$ is the quotient field of $R$. Then $\bar{A}^{F}$ is a finite $A$-module (and is therefore finitely generated over $R$ ).

We remind the reader of the definition of a restricted domain:

Definition 1.3. A domain (i.e., an integral domain) $R$ is said to be a restricted domain if $R$ is Noetherian and for every finite algebraic field extension $F$ of the

Received by the editors August 28, 2006.

2000 Mathematics Subject Classification. Primary 14A05.

Key words and phrases. Commutative algebra.

The first author's work was partly supported by NSF Grant DMS 99-88166 and NSA Grant MSP H98230-05-1-0040. 
quotient field $k$ of $R$ we have that $\bar{R}^{F}$ is a finite $R$-module. Recall that an affine domain over a domain $R$ is an overdomain $A$ of $R$ such that $A$ is a finitely generated ring extension of $R$. Thus Claim 1.2 may be paraphrased by saying that an affine domain over a restricted domain is again a restricted domain.

The offered proof of Claim 1.2 in [6] depends heavily on the false Claim 1.1. In Example 3.5 of Section 3 we show that Claim 1.2 is indeed false. Our very special thanks to William Heinzer for suggesting a source for this counterexample.

\section{First counterexample}

Example 2.1. This counterexample to Claim 1.1 appears in 1 on page 592. Let us assume that Claim 1.1 is true. Take $R=\mathbb{Z}$ and let $X_{1}, \ldots, X_{d}$ be indeterminates over $\mathbb{Z}$. Let $A=R\left[x_{1}, \ldots, x_{n}\right]$ with $n=d+1$ and $x_{i}=X_{i}$ for $1 \leq i \leq d$. Let $x_{n} \in \mathbb{Q} \backslash \mathbb{Z}$, e.g., $x_{n}=\frac{1}{2}$. By the claim we find $y_{1}, \ldots, y_{d}$ in $A$ such that $A$ is integral over $B=R\left[y_{1}, \ldots, y_{d}\right]$. Now $y_{1}, \ldots, y_{d}$ are algebraically independent over $\mathbb{Q}$, so $B$ is a normal domain. Hence the minimal polynomial of $x_{n}$ over the quotient field of $B$ belongs to $B[Y]$, where $Y$ is an indeterminate. But clearly this polynomial is $Y-x_{n}$, and we have a contradiction.

\section{SECOnd COUntereXample}

For this counterexample, we will use the theorems of Rees stated in the second paragraph of [4, but first we require a definition.

Definition 3.1. Let $R$ be a local ring. $R$ is said to be analytically unramified if the completion $\widehat{R}$ of $R$ contains no nonzero nilpotent element. We say that $R$ is analytically ramified if this condition does not hold.

We may now formulate the theorem of Rees as:

Theorem 3.2. Let $R$ be a local ring such that $R$ has no nonzero nilpotent element. Then $R$ is analytically unramified if and only if for every finite sequence of elements $x_{1}, \ldots, x_{n}$ in the total quotient ring $k$ of $R$, upon letting $A=R\left[x_{1}, \ldots, x_{n}\right]$, we have that $\bar{A}^{k}$ is a finite A-module.

In order to proceed, we must remind the reader of another definition:

Definition 3.3. Given any ring $R$, clearly there exists a unique homomorphism $\mathbb{Z} \rightarrow R$. The unique nonnegative generator of the kernel of this homomorphism is the characteristic of $R$. The image of this homomorphism is called the prime ring of $R$.

Now we wish to construct a counterexample to Zariski-Samuel's second claim. We would like to find a normal local domain containing a field of characteristic 0 whose completion has nonzero nilpotent elements. This can be done by a theorem of Heitmann, specifically Theorem 8 in [2], which is as follows:

Theorem 3.4. Let $T$ be a complete local ring of dimension at least 2 such that no nonzero element of its prime ring is a zerodivisor in $T$. Then $T$ is the completion of a local unique factorization domain $R$.

Example 3.5. Let us take $T=\kappa[[X, Y, Z]] /\left(Z^{2}\right)$, where $\kappa$ is a field of characteristic 0 . Then the prime ring of $T$ is $\mathbb{Z}$, and no nonzero element of $\mathbb{Z}$ is a zerodivisor in 
$T$. Also $T$ is a homomorphic image of a power series ring, so it is a complete local ring, and clearly its dimension is 2. So $T$ satisfies the hypotheses of Heitmann's theorem, and hence $T$ is the completion of a local unique factorization domain $R$. Now $R$ is a normal Noetherian domain of characteristic 0 and hence, by a standard theorem, $R$ is a restricted domain; for instance see Theorem (T141) on page 522 of [1. However, the image of $Z$ is a nonzero nilpotent in $T$, so $R$ is analytically ramified. Therefore by Rees' theorem we can find a finite sequence of elements $x_{1}, \ldots, x_{n}$ in the quotient field $k$ of $R$ such that upon letting $A=R\left[x_{1}, \ldots, x_{n}\right]$ we have that $\bar{A}^{k}$ is not a finite $A$-module. Since $k$ is certainly a finite algebraic extension of itself, and $A$ is an affine domain over $R$, we have thus constructed our counterexample.

Remark 3.6. The fact that Claim 1.2 is true when $R$ is a field is proved in Theorem (T145) on page 530 of [1]. In (E28) on page 592 of [1] it was questioned whether Claim 1.2 is true as it stands, and this inspired the desire to search for our Example 3.5. Nagata, in (36.1) on page 131 of [3], defines a pseudogeometric ring to be a Noetherian ring $R$ such that every domain, which is a homomorphic image of $R$, is a restricted domain. In (36.5) on page 132 of [3], he proves that every finitely generated ring extension of a pseudogeometric ring is again pseudogeometric.

\section{REFERENCES}

1. S. S. Abhyankar, Lectures on Algebra, Volume I, World Scientific, Hackensack, NJ, 2006.

2. R. C. Heitmann, Characterization of Completions of Unique Factorization Domains, Trans. Amer. Math. Soc., vol. 337, no. 1, Amer. Math. Soc. (May, 1993), Providence, RI, pp. 379-387. MR 1102888 (93g:13006)

3. M. Nagata, Local Rings, Interscience Publishers, New York, 1962. MR0155856 (27:5790)

4. D. Rees, A Note on Analytically Unramified Local Rings, Journal London Math. Soc., vol. 36, London Math. Soc. (1961), pp. 24-28. MR0126465 (23:A3761)

5. O. Zariski and P. Samuel, Commutative Algebra, Volume I, Springer-Verlag, New York, 1960.

6. O. Zariski and P. Samuel, Commutative Algebra, Volume II, Springer-Verlag, New York, 1960. MR0120249(22:11006)

Department of Mathematics, Purdue University, West Lafayette, Indiana 47907

E-mail address: ram@cs.purdue.edu

Department of Mathematics, Purdue University, West Lafayette, Indiana 47907

E-mail address: bkravitz@math.purdue.edu 